\title{
Overexpression of Annexin A1 protects against benzo[a]pyrene-induced bronchial epithelium injury
}

\author{
YANFEI CUI and SHENGYA YANG
}

\begin{abstract}
Department of Tuberculosis, Hangzhou Red Cross Hospital/Zhejiang Chinese Medicine and
Western Medicine Integrated Hospital, Hangzhou, Zhejiang 310003, P.R. China
\end{abstract}

Received January 19, 2018; Accepted April 24, 2018

DOI: $10.3892 / \mathrm{mmr} .2018 .8998$

\begin{abstract}
The incidence of asthma is increasing worldwide. Bronchial epithelium injury is common in asthma. The regulatory role of Annexin A1 (ANXA1) in bronchial epithelium injury is currently not well understood. The aim of the present study was to evaluate the role of ANXA1 on bronchial epithelium injury. The cell viability and levels of apoptosis were respectively tested by Cell Counting Kit- 8 and flow cytometry. Reactive oxygen species (ROS) content and the activity of oxidative indicators were assessed by commercial kits. Enzyme linked immunosorbent assay was performed to detect the activity of active caspase-3. Reverse transcription-quantitative polymerase chain reaction and western blot assays were used to determine the expression levels of the target factors. The results demonstrated that ANXA1 improved the viability of benzo[a]pyrene (Bap)-treated bronchial epithelial cells. The Bap-induced oxidative stress was mitigated by the reduction in ROS generation, and the regulation of the activity of superoxide dismutase, glutathione peroxidases, malondialdehyde and lactic dehydrogenase. In addition, apoptosis was decreased by ANXA1 via the reduction of the expression of B-cell lymphoma 2 (Bcl-2), and the increase in the expression of $\mathrm{Bcl}-2$-associated $\mathrm{X}$ protein and cyclin D1. Furthermore, the expression of phosphatase and tensin homolog (PTEN) and focal adhesion kinase (FAK) was rescued and the phosphorylation of phosphatidylinositol 3-kinase (PI3K)/protein kinase B (Akt) was depressed by ANXA1, when compared with the Bap group. SF1670 treatment reversed the anti-apoptotic effect of ANXA1. In conclusion, the results highlighted the protective effects of ANXA1 on bronchial epithelium injury, which most likely occurred via the PTEN/FAK/PI3K/Akt signaling pathway.
\end{abstract}

Correspondence to: Dr Shengya Yang, Department of Tuberculosis, Hangzhou Red Cross Hospital/Zhejiang Chinese Medicine and Western Medicine Integrated Hospital, 208 East Huancheng Road, Hangzhou, Zhejiang 310003, P.R. China E-mail: shengya_yang345sy@163.com

Key words: Annexin A1, oxidative stress, apoptosis, bronchial epithelium injury
Thus, the present study contributes to a potential therapeutic strategy for asthma patients.

\section{Introduction}

As a major public health problem, the incidence of asthma is on the rise worldwide $(1,2)$. This condition is accompanied by airflow obstruction. Airway remodelling is one of the major characteristics of asthma, and has attracted many attentions due to its critical role in asthma disease (3). Bronchial epithelium, as a physical mediator between the internal milieu and the external environment, is exposed to many environmental factors (i.e. air pollutants, allergens and environmental chemicals), therefore leading remodelling, which is considered as a response to cell and tissue damage $(4,5)$. Many efforts have been done to combat asthma, however, only a few strategies proved to be effective in preventing/alleviating this condition. Therefore, it is necessary to develop new methods for asthma treatment. Benzo[a]pyrene (Bap) is a common environmental chemical carcinogen, which has strong carcinogenicity, mutagenicity and teratogenicity (6). The atmospheric Bap mainly comes from wood burning (7). Lung epidermal cells are the main media, via which some harmful substances are absorbed by human bodies. Research reported that Bap is able to induce pulmonary epithelial injury (8). Thus, Bap will be used to help set up the bronchial epithelium injury model in the present study.

The balance of redox state is considered to contribute to multiple cellular events (9). Emerging evidences have suggested that reactive oxygen species (ROS) are the mediator for the chronic airway diseases, which includes asthma $(10,11)$. ROS is a byproduct of cell respiration, and it can be removed by antioxidant enzymes under normal conditions. However, if the oxidant/antioxidant balance is disrupted, excessive ROS oxidation metabolites will accumulate in cells, and therefore inducing oxidative stress and creating impairments to the cells (12). The predominant consequence of oxidative stress is the cell apoptosis (13). Thus, strategies targeting at the oxidative stress and apoptosis of bronchial epithelial cells may be effective to control tissue injury in the treatment of asthma.

Various molecules and factors, such as phosphatase and tensin homolog (PTEN), focal adhesion kinase (FAK) and phosphatidylinositol 3-kinase (PI3K)/Akt, are associated with cell survival $(14,15)$. PTEN has generally been recognized as a tumor suppressor (16), and it may be a gatekeeper in 
acute lung injury and fibrosis (17). Moreover, PI3K/Akt, as an important signaling pathway that regulates cell survival, is possibly negatively regulated by PTEN (18). Furthermore, FAK is critical to the integration of various signals (19), which are of significance to the cell survival and apoptosis $(15,20)$. FAK is reported to be the upstream of PI3K/Akt signaling pathway as well (21). Therefore, these signals may be the possible molecule targets in the treatment of bronchial epithelial cell injury.

Annexin A1 (ANXA1), which originally believed to inhibit phospholipase activity (22), has been reported to be able to modulate various cellular functions in multiple cell types $(23,24)$. Protective roles of ANXA1 have been observed $(25,26)$. A recent study demonstrated that ANXA1 is able to accelerate the healing of gastric ulcers that caused by indomethacin (27). However, to the best of our knowledge, the regulatory role of ANXA1 in bronchial epithelium injury and the potential mechanisms have not been validated. Thus, the main scope of this study was to determine the effect(s) of ANXA1 on bronchial epithelial cell injury in vitro.

\section{Materials and methods}

Cell culture and cell viability assay. Human bronchial epithelial cells BEAS-2B (CRL-9609; ATCC, Manassas, VA, USA) (passage numbers 60-80) were maintained in Dulbecco's Modified Eagle's Medium (DMEM; Thermo Fisher Scientific, Inc., Waltham, MA, USA) that containing with $10 \%$ fetal bovine serum, and, $1 \%$ streptomycin/penicillin (Gibco; Thermo Fisher Scientific, Inc.) at $37^{\circ} \mathrm{C}$ with and $5 \% \mathrm{CO}_{2}$ at $37^{\circ} \mathrm{C}$. CCK-8 method (Beyotime Institute of Biotechnology, Haimen, China) was adopted to measure the cell viability. The cells were seeded at a density of $2.5 \times 10^{3}$ cell/well. After $24 \mathrm{~h}, 10 \mu \mathrm{l} \mathrm{CCK}-8$ solutions were added into the incubator and then maintained with the cells at $37^{\circ} \mathrm{C}$ for $4 \mathrm{~h}$. The absorbance $450 \mathrm{~nm}$ was measured by a microplate reader (Bio-Rad Laboratories, Inc., Hercules, CA, USA).

Cell grouping and transfection. The cell grouping were as follows: i) Control group, normal cells; ii) empty vector group, cells that were transfected with negative vectors; iii) NXA1 group: cells that were transfected with ANXA1 over-expression vectors; iv) Bap group, cells that were treated with Bap $(64 \mu \mathrm{M}$ for $6 \mathrm{~h}$, as stated in the results section); v) emp+Bap, Cells that were transfected with negative vectors, and then treated with $64 \mu \mathrm{M}$ Bap for $6 \mathrm{~h}$ ), Anx+Bap: Cells that were transfected with ANXA1 vector and then treated with $64 \mu \mathrm{M}$ Bap for 6 h. E.V.=empty vector, Bap=benzo[a]pyrene. Lipofectamine LTX $^{\mathrm{TM}}$ Reagent (Invitrogen; Thermo Fisher Scientific, Inc.) was used for cell transfection. The p-Receiver-M01/ANXA1 vector for over-expression were ordered from GeneCopoeia (Rockville, MD, USA). The expression vector $\mathrm{p}$-Receiver-M01 served as negative control vector. The cells were cultured in serum-free medium overnight. Lipofectamine Reagent and DNA solution (vector ANXA1 or negative control) were gently mixed. After being incubation for $30 \mathrm{~min}$, the Lipofectamine-DNA complex was added into cells, and mixed by rocking the plate back and forth. Subsequently, the plate was incubated in an incubator. Then the cells in each group were treated with or without Bap. The cells were pretreated

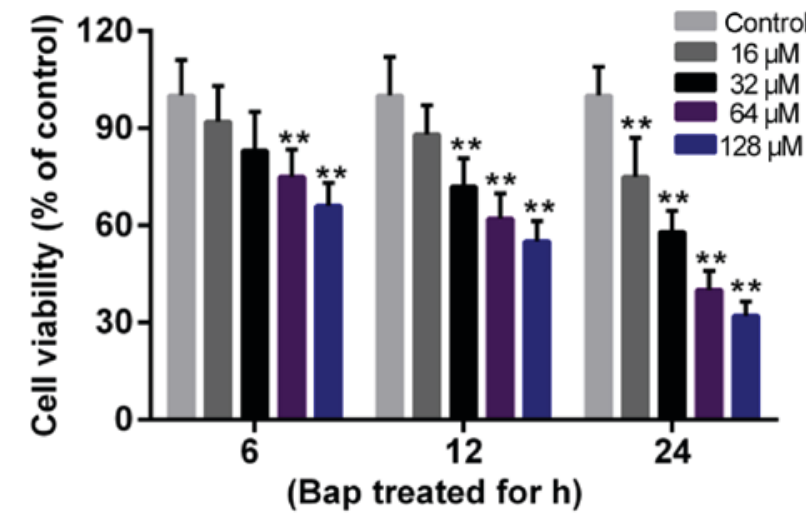

Figure 1. Cytotoxicity of Bap to bronchial epithelial cells. Cells were incubated with different concentrations of $\operatorname{Bap}(16,32,64$ and $128 \mu \mathrm{M})$. The cell viability was detected at 6,12 and $24 \mathrm{~h}$ following incubation with Cell Counting Kit- 8 reagent. ${ }^{* *} \mathrm{P}<0.01$ vs. control group. Bap, benzo[a]pyrene.

with 500 nM SF1670 (MedchemExpress, South Brunswick, $\mathrm{NJ}, \mathrm{USA}$ ) at $37^{\circ} \mathrm{C}$ for $30 \mathrm{~min}$ to inhibit the PTEN pathway, following steps described in a previous study (28).

Apoptosis estimation. Flow cytometry (FCM) assay was performed to assess cell apoptosis using FITC/Annexin V Apoptosis detection kit I (BD Biosciences, Franklin Lakes, NJ, USA), according to the manufacturer's instructions. The cells were incubated with FITC/Annexin V and PI at room temperature in the dark for cellstaining. The cells were fixed with paraformaldehyde $(0.5 \%)$, and then they were loaded on a FACStar Plus flow cytometer (BD Biosciences).

ROS content detection. To evaluate the intracellular ROS levels, the cells were stained with $10 \mu \mathrm{M}$ fluorescent dye 2,7-dichlorofluorescein diacetate (DCFH-DA) dye (Sigma-Aldrich; Merck KGaA, Darmstadt, Germany) at $37^{\circ} \mathrm{C}$ for $15 \mathrm{~min}$ in the dark. DCFH-DA can be transformed into fluorescent DCF by the presence of ROS. The fluorescent intensity indicated the ROS levels. The DCF fluorescence signals were detected by a fluorescence plate reader (BioTek Instruments, Inc., Winooski, VT, USA).

Oxidative stress measurement. The cells were centrifuged and the supernatant was collected. Following the manufacturer's instructions, the activities of superoxide dismutase (SOD; S0101), glutathione peroxidases (GPX; S0056), malondialdehyde (MDA; S0131) and lactic dehydrogenase (LDH; C0016) were assessed by available commercial kits (Beyotime Institute of Biotechnology). A microplate reader (Bio-Rad Laboratories, Inc.) was used to measure the absorbance when appropriate.

Enzyme linked immunosorbent assay (ELISA). Active Caspase-3 Quantikine ELISA kit (R\&D Systems, Inc., Minneapolis, MN, USA) was used to detect the activity of active caspase-3. To explain further, the cells were collected and re-suspended. Then, the samples were added into each well and then incubated at room temperature for $1 \mathrm{~h}$. After being washed, the substrate solution was added into the wells and then incubated for $30 \mathrm{~min}$ at room temperature. Finally, 
A

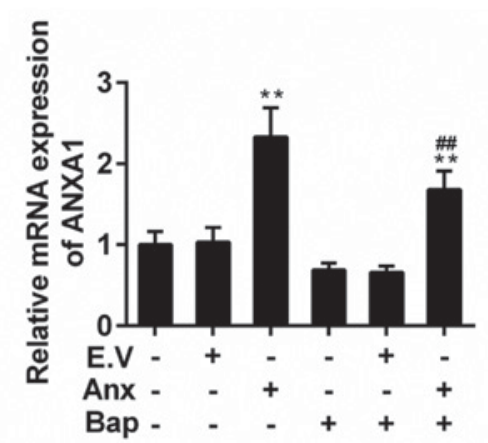

C

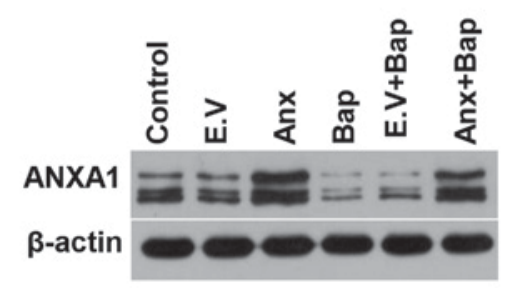

B

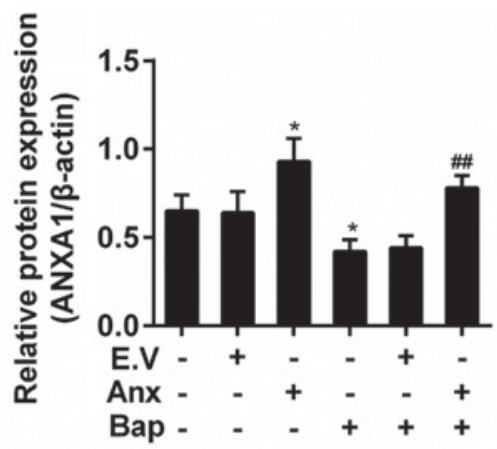

D

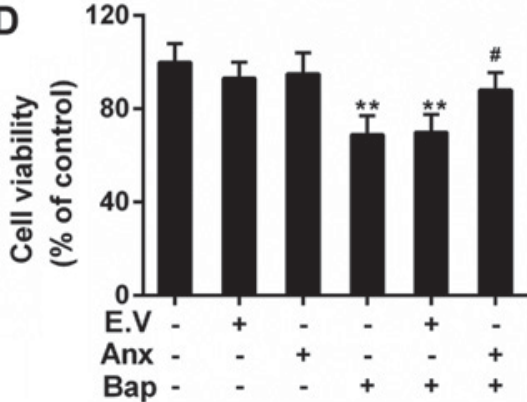

Figure 2. Effect of ANXA1 on the proliferation of BEC. (A) Reverse transcription-quantitative polymerase chain reaction was used to test the mRNA expression of ANXA1. (B) The protein expression of ANXA1 was determined by (C) western blotting. (D) Cell Counting Kit-8 assay was performed to analyze the effect of ANXA1 on BEC. The cells were treated with $64 \mu \mathrm{M}$ Bap for $6 \mathrm{~h}$ to establish the cell injury model. ${ }^{*} \mathrm{P}<0.05$ and ${ }^{* *} \mathrm{P}<0.01$ vs. control group; ${ }^{*} \mathrm{P}<0.05$ and ${ }^{\# \#} \mathrm{P}<0.01$ vs. E.V.+Bap group. ANXA1/Anx, Annexin A1; BEC, bronchial epithelial cells; Bap, benzo[a]pyrene; E.V., empty vector.
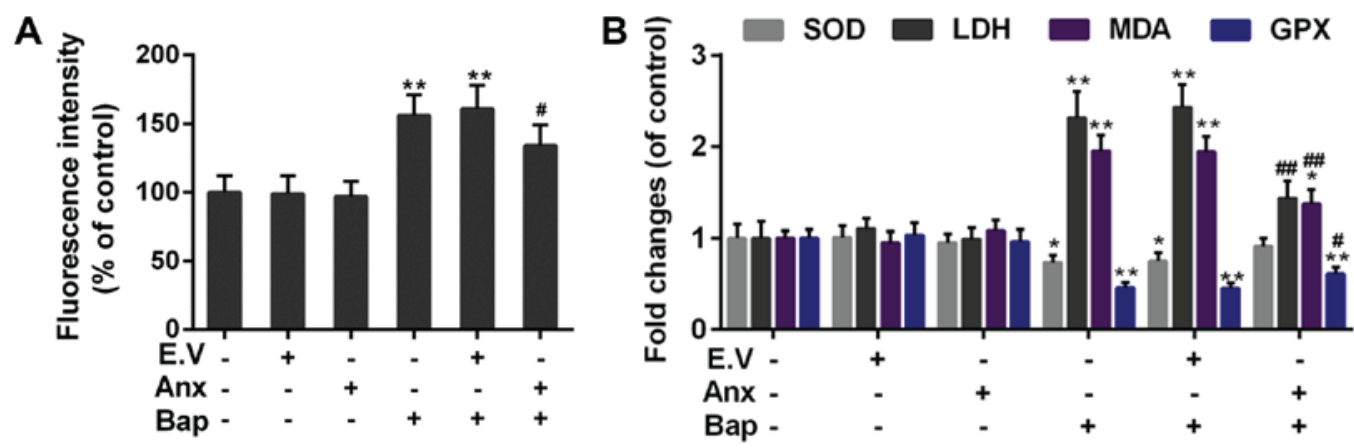

Figure 3. Effect of ANXA1 on oxidative stress. (A) The ROS content was determined by 2,7-dichlorofluorescein diacetate dye. (B) The activities of SOD, GPX, MDA and LDH were regulated by ANXA1. The cells were treated with $64 \mu \mathrm{M}$ Bap for $6 \mathrm{~h}$ to establish the cell injury model. ${ }^{*} \mathrm{P}<0.05$ and ${ }^{* *} \mathrm{P}<0.01 \mathrm{vs}$. control group; ${ }^{\# P}<0.05$ and ${ }^{\# \#} \mathrm{P}<0.01$ vs. E.V.+Bap group. ANXA1/Anx, Annexin A1; ROS, reactive oxygen species; SOD, superoxide dismutase; GPX, glutathione peroxidases; MDA, malondialdehyde; LDH, lactic dehydrogenase; Bap, benzo[a]pyrene; E.V., empty vector.

the stop solution was added into each well. The absorbance was read at $450 \mathrm{~nm}$ using a microplate reader (Bio-Rad Laboratories, Inc.).

Reverse transcription-quantitative polymerase chain reaction $(R T-q P C R)$ analysis. Total RNA was isolated from the harvested cells using TRizol regent (Invitrogen; Thermo Fisher Scientific, Inc.), following the manufacturer's protocols. The integrity of RNA was tested by agarose gel electrophoresis. The total RNA $(1 \mu \mathrm{g})$ was reverse-transcribed into cDNA using M-MLV (Promega Corporation, Madison, WI, USA). RT-qPCR analysis was performed to detect the relative expression of target genes. The amplification was conducted on ABI Prism 7,500 Sequence detection instrument using SYBR Premix TaqTM II kit (Takara Bio, Inc., Otsu, Japan).
The $2^{-\Delta \Delta C q}$ method was used for expression quantification (29). The primers used were as follows: Cyclin D1 forward: 5'-CCC TCGGTGTCCTACTTCAA-3'; Cyclin D1 reverse: 5'-CTT AGAGGCCACGAACATGC-3'; B-cell lymphoma 2 (Bcl-2) forward: 5'-CACACACACACATTCAGGCA-3'; Bcl-2 reverse: 5'-GGCAATTCCTGGTTCGGTTT-3'; Bcl-2-associated X protein (Bax) forward: 5'-TGGCCTCCTTTCCTACTTCG-3'; Bax reverse: 5'-AAAATGCCTTTCCCCGTTCC-3'; $\beta$-actin forward: 5'CCCGCGAGCACAGCTTCTTTG3'; $\beta$-actin reverse: 5'ACATGCCGGAGCCGTTGTCGAC3'.

Western blot analysis. The lysed cells were centrifuged at $12,000 \mathrm{~g}$ at $4^{\circ} \mathrm{C}$ for $10 \mathrm{~min}$. The protein concentration was determined by BCA Protein Assay Kit (Bio-Rad Laboratories, Inc.). The proteins were denatured by being heated in boiling water for 

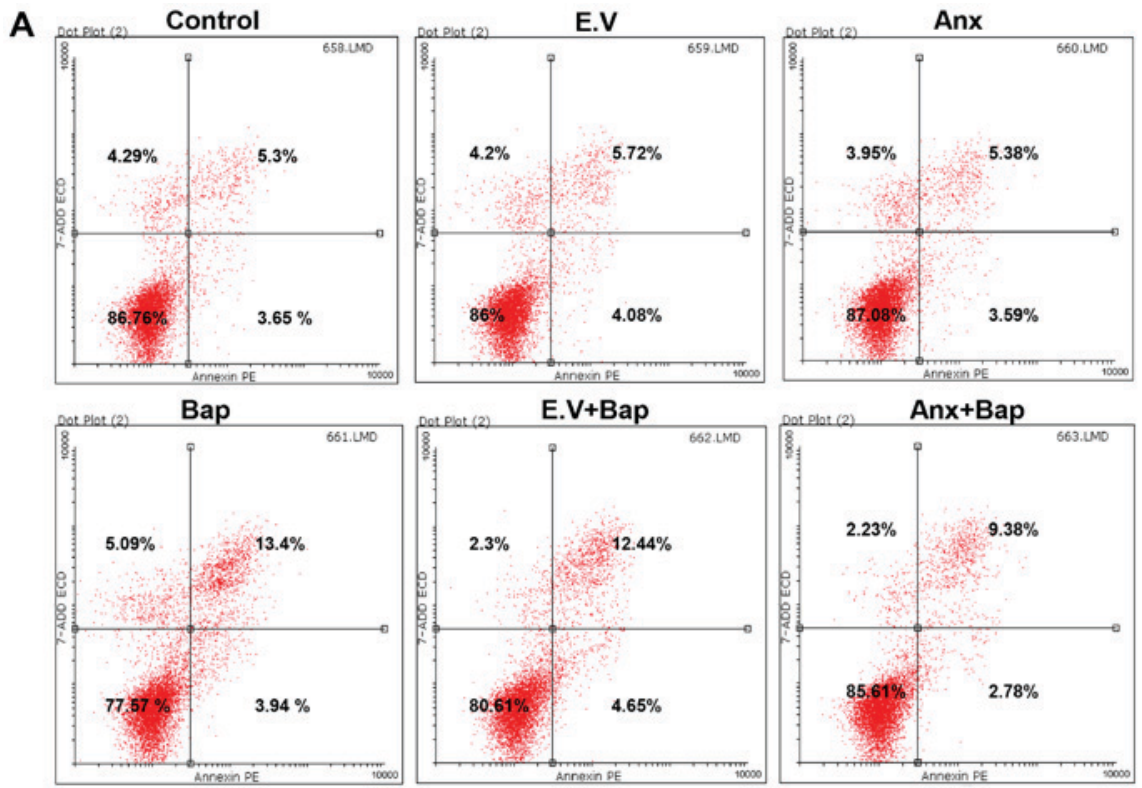

B

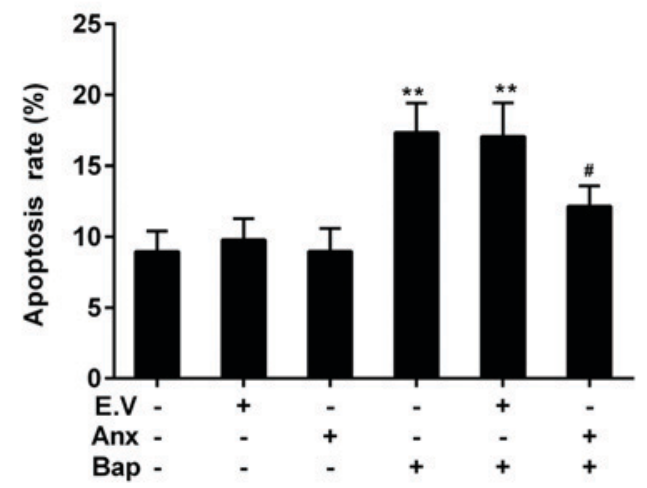

Figure 4. Effect of ANXA1 on apoptosis. (A) The levels of apoptosis was estimated by FCM assay. (B) The apoptosis levels were calculated based on FCM data. The cells were treated with $64 \mu \mathrm{M}$ Bap for $6 \mathrm{~h}$ to establish the cell injury model. ${ }^{* *} \mathrm{P}<0.01$ vs. control group; "P<0.05 vs. E.V.+Bap group. ANXA1/Anx, Annexin A1; FCM, flow cytometry; Bap, benzo[a]pyrene; E.V., empty vector.

5 min. The proteins were then separated on SDS-PAGE gel by electrophoresis. After being transferred onto PVDF membrane, the proteins were blocked with skimm milk for $2 \mathrm{~h}$ at room temperature. Primary antibodies were incubated with PVDF membrane overnight at $4^{\circ} \mathrm{C}$. Information of primary antibodies used in the experiment was as follows: Anti-ANXA1 (ab19830, 1:2,000), anti-Cyclin D1 (ab134175, 1:10,000), anti-Bax (ab32503, 1:1,000), anti-Bcl-2 (ab692, 1:500), anti-PTEN (ab170941, 1:4,000), anti-FAK (ab76496, 1:1,000), anti-Akt1/2 (ab182729, 1:5,000), anti-p-Akt (ser473) (ab81283, 1:5,000; all from Abcam, Cambridge, UK), PI3Kinase Class III (4263, 1:1,000; CST Biological Reagents Co.,Ltd., Shanghai,China), anti-p-PI3Kinase Class III (Ser249) (13857, 1:1,000; CST Biological Reagents Co., Ltd.) and anti- $\beta$-actin (ab8226, 1:5,000; Abcam). Subsequently, the secondary antibodies (ab205718, 1:5,000; Abcam) coupled with horseradish peroxidase were added and interact with the primary antibodies. The band was developed with enhanced chemiluminescence system (GE Healthcare, Chicago, IL, USA). The grey value was read using quantity one 4.6.2.

Statistical analysis. Data are presented as mean \pm standard deviation. Student's t-test or one-way analysis of variance followed by Dunnett's post hoc test was performed to compare the differences among groups by using GraphPad Prism Software 6 (GraphPad Software, Inc., La Jolla, CA, USA), when appropriate. $\mathrm{P}<0.05$ was considered to indicate a statistically significant difference.

\section{Results}

ANXA1 improved the viability of Bap-treated bronchial epithelial cells. The effect of Bap on cell viability was determined first. Data showed that the cell viability deceased gradually with the increase of time and of the dosage of Bap. The viability began to decline significantly after $6 \mathrm{~h}$ of $64 \mu \mathrm{M}$ Bap incubation (Fig. 1). Thus, incubating $64 \mu \mathrm{M}$ Bap for $6 \mathrm{~h}$ was selected for inducing bronchial epithelium injury. Moreover, the expression of ANXA1 was depressed by the presence of Bap in mRNA and protein levels. However, we observed that the over-expression of ANXA1 reversed this phenomenon (Fig. 2A-C). Furthermore, the decreased viability of bronchial epithelial cells was recovered by ANXA1 over-expression (Fig. 2D).

ANXA1 reduced the Bap-mediated oxidative stress in bronchial epithelial cells. ROS induction is a critical mechanism of 
A

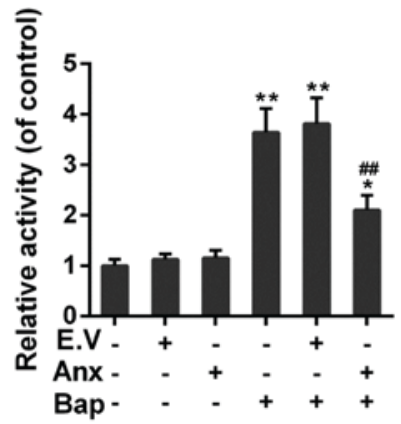

C

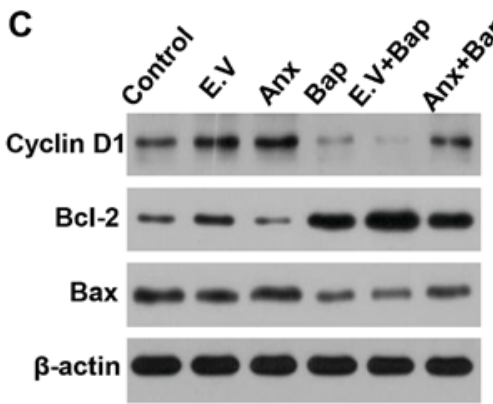

B
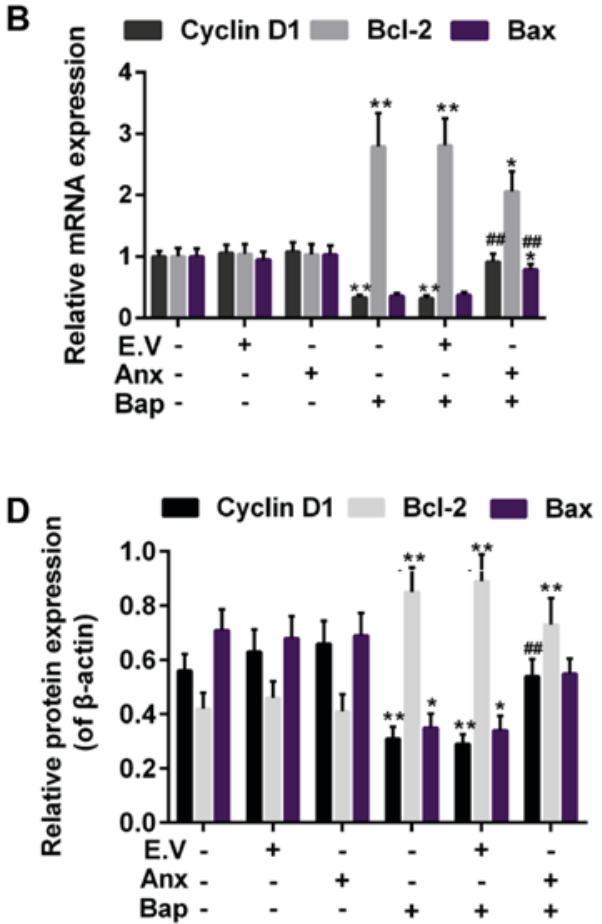

Figure 5. Effect of ANXA1 on apoptosis-associated proteins. (A) The activity of active caspase-3 was assessed by ELISA. (B) Reverse transcription-quantitative polymerase chain reaction was used to test the mRNA expressions of cyclin D1, Bax and Bcl-2. (C) Western blotting was performed to (D) evaluate the protein expressions of cyclin D1, Bax and Bcl-2. The cells were treated with $64 \mu \mathrm{M}$ Bap for $6 \mathrm{~h}$ to establish the cell injury model. ${ }^{*} \mathrm{P}<0.05$ and ${ }^{* *} \mathrm{P}<0.01$ vs. control group; ${ }^{\# \#} \mathrm{P}<0.01$ vs. E.V.+Bap group. ANXA1/Anx, Annexin A1; Bax, Bcl-2-associated X protein; Bcl-2, B-cell lymphoma 2; Bap, benzo[a]pyrene; E.V., empty vector.

A

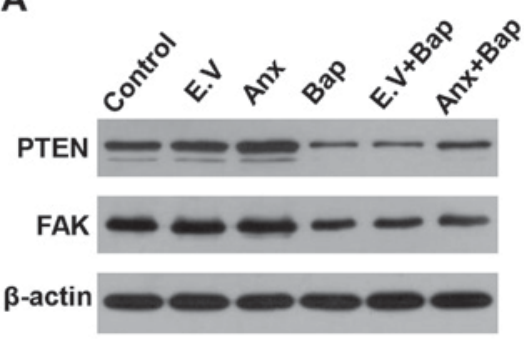

C

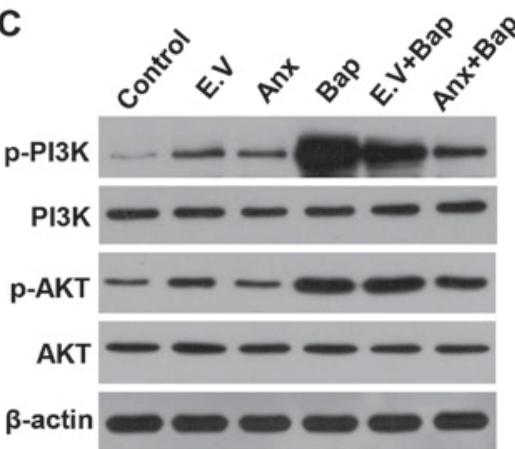

B

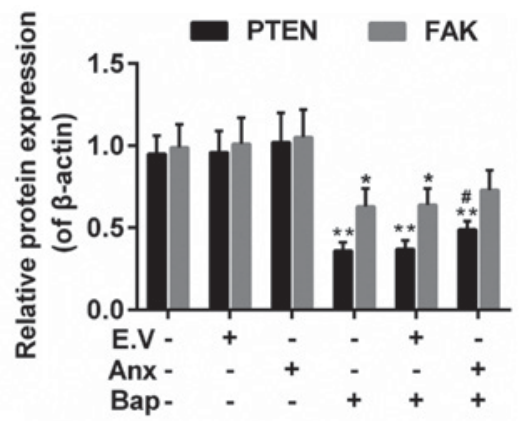

D

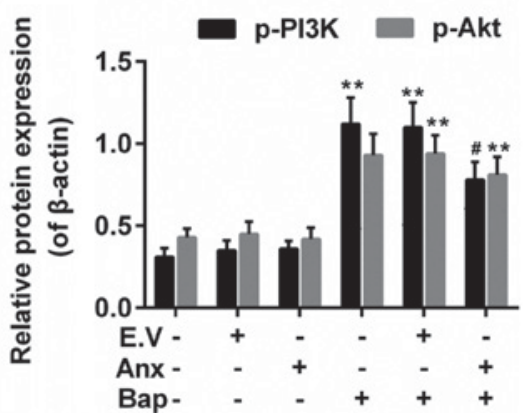

Figure 6. Effect of ANXA1 on the activities of PTEN, FAK and PI3K/Akt. (A) Western blotting was used to determine the protein levels of (B) PTEN and FAK (C) Western blotting was also performed to determine the protein levels of (D) PI3K/Akt, p-PI3K and p-Akt. The cells were treated with $64 \mu \mathrm{M}$ Bap for $6 \mathrm{~h}$ to establish the cell injury model. ${ }^{*} \mathrm{P}<0.05$ and ${ }^{* *} \mathrm{P}<0.01$ vs. control group; ${ }^{"} \mathrm{P}<0.05$ vs. E.V.+Bap group. ANXA1/Anx, Annexin A1; PTEN, phosphatase and tensin homolog; FAK, focal adhesion kinase; PI3K, phosphatidylinositol 3-kinase; Akt, protein kinase B; p-, phosphorylated; Bap, benzo[a]pyrene; E.V., empty vector.

bronchial epithelium injury (30). The effect of ANXA1 on ROS generation was tested. Our data indicated that the intracellular ROS content was mitigated in ANXA1+Bap group, compared to Bap group (Fig. 3A). Furthermore, compared to Bap group, the activity of free radical scavenging enzymes, including superoxide dismutase (SOD) and glutathione peroxidase (Gpx) 
A
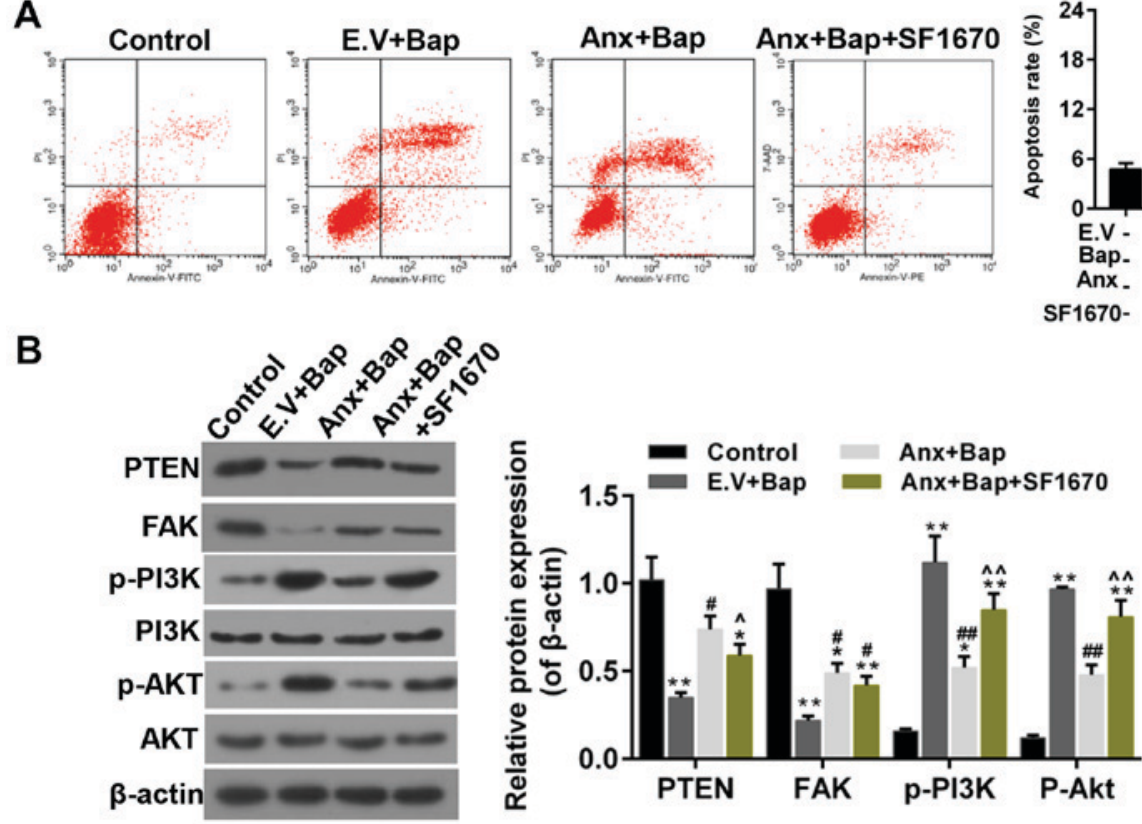

Figure 7. SF1670 reverses the effect of ANXA1. (A) The levels of apoptosis were estimated by flow cytometry assay. (B) Western blotting was performed to determine the expressions of PTEN, FAK, PI3K/Akt, p-PI3K and p-Akt. The cells were treated with $64 \mu \mathrm{M}$ Bap for $6 \mathrm{~h}$ to establish the cell injury model. ${ }^{*} \mathrm{P}<0.05$ and ${ }^{* *} \mathrm{P}<0.01$ vs. control group; ${ }^{\#} \mathrm{P}<0.05$ and ${ }^{\# \#} \mathrm{P}<0.01$ vs. E.V.+Bap group; $\wedge \mathrm{P}<0.05, \wedge \wedge \mathrm{P}<0.01$ vs. ANXA1+BaP group. ANXA1/Anx, Annexin A1; PTEN, phosphatase and tensin homolog; FAK, focal adhesion kinase; PI3K, phosphatidylinositol 3-kinase; Akt, protein kinase B; p-, phosphorylated; Bap, benzo[a]pyrene; E.V., empty vector.

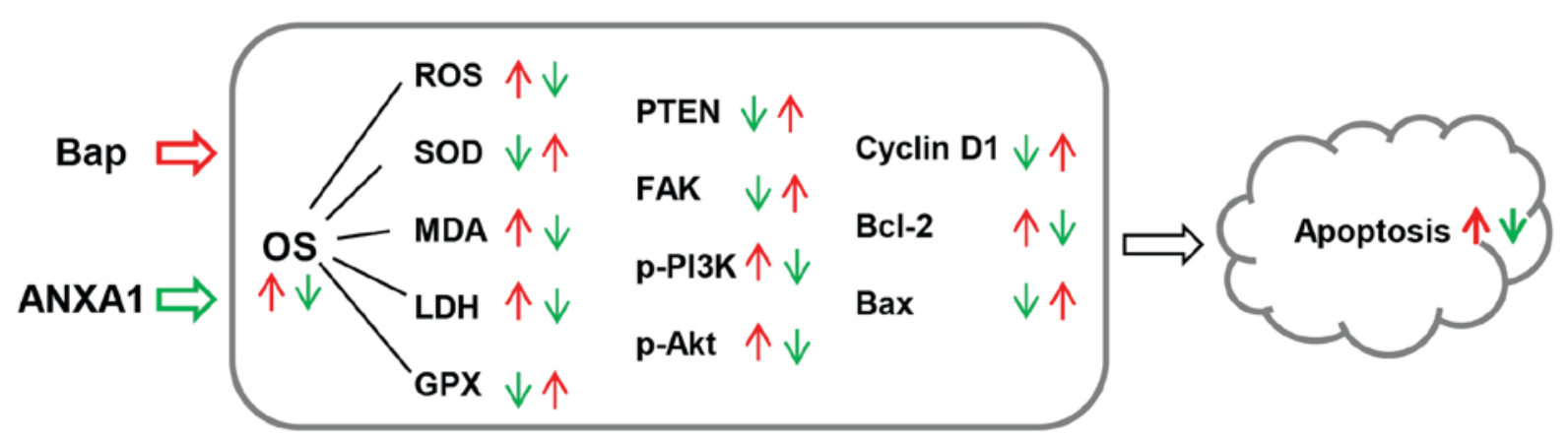

Figure 8. The model applied in the present study. Red arrows indicate the effect of Bap, and green arrows indicate the effect of ANXA1. Bap, benzo[a]pyrene; OS, oxidative stress; ANXA1, Annexin A1; PTEN, phosphatase and tensin homolog; FAK, focal adhesion kinase; PI3K, phosphatidylinositol 3-kinase; Akt, protein kinase B; p-, phosphorylated; SOD, superoxide dismutase; GPX, glutathione peroxidases; MDA, malondialdehyde; LDH, lactic dehydrogenase; ROS, reactive oxygen species; Bcl-2, B-cell lymphoma 2; Bax, Bcl-2-associated X protein.

was rescued. however, the content of oxidative injury makers, including malondialdehyde (MDA) and lactate dehydrogenase (LDH), was declined by ANXA1 over-expression (Fig. 3B).

ANXA1 depressed the Bap-mediated apoptosis in bronchial epithelial cells. Apoptosis can be triggered by oxidative stress (31). FCM data showed that cell apoptosis level was lower in ANXA1+Bap group than that in Bap group (Fig. 4A and B). Moreover, the activity of caspase- 3 was reduced by the over-expression of ANXA1 (Fig. 5A). In addition, Bcl-2 and Bax, as members of the Bcl-2 family, is pro-apoptotic and anti-apoptotic apoptosis proteins, respectively (32). Cyclin D1, as a cell cycle regulator, is active in cell apoptosis pathways (33). Thus, the anti-apoptotic effect of ANXA1 was further determined by testing the expression of these apoptosis-related genes. Our data recorded that the increase expression of $\mathrm{Bcl}-2$ was alleviated, whereas the expression of Bax and cyclin D1 was rescued by the over-expression of ANXA1 in both mRNA and protein levels, compared to Bap group (Fig. 5B-D).

ANXA1 modulated the activity of PTEN/FAK/PI3K/Akt signals.PTEN/PI3K/Akt is reported to be related to the lung cell injury $(34,35)$. And FAK is associated with cell apoptosis (36). Thus, the effect of ANXA1 on these factors was investigated. We observed that Bap reduced expression of PTEN and FAK, which was recovered by ANXA1 (Fig. 6A and B). Moreover, the results showed that the enhanced levels of phosphorylation of PI3K and Akt (p-PI3K/p-Akt) induced by Bap were partly declined by ANXA1 (Fig. 6C and D).

Inhibition of PTEN reversed the anti-apoptotic effect of ANXA1. Subsequently, an inhibitor of PTEN-SF1670, 
was adopted to further confirm the role of PTEN in the anti-apoptotic effect that conferred by ANXA1. The results showed that the apoptosis mediated by Bap was mitigated by over-expression of ANXA1, whereas SF1670 reversed the anti-apoptotic effect of ANXA1 (Fig. 7A). Moreover, the WB data indicated that the enhanced expression of PTEN and FAK was decreased by SF1670. And the decreased expression of p-PI3K/p-Akt was augmented by SF1670 (Fig. 7B). The work model relate to the current study was shown in Fig. 8.

\section{Discussion}

In environmental pollutants and cigarette smoke, many toxic agents, of which Bap is a type, produce harmful effects on human beings, especially on the respiratory tract $(37,38)$. Bap was employed to induce the bronchial epithelium injury in this study. Consistent with the previous study, it was obvious that the cytotoxic effect of Bap was augmented with the increasing of concentration and the progress of incubation time. This study investigated oxidative stress and apoptosis in human bronchial epithelial cells during the incubation with Bap and the expression of AnxA1. The data suggested that the ANXA1 exerted a protective role in Bap-induced bronchial epithelium injury. This outcome was in line with the increased histopathologic mucosal injury exhibited in AnxA1-deficient animals (39).

Several mechanisms have been put forward to interrupt the cell injury in airways, among which intracellular induction of ROS is a type. ROS are molecules, which have a strong cytotoxic capacity. Oxidative stress occurs when the ROS accumulate in living cells (12). Our data showed that the incubation of Bap remarkably increased the ROS formation in bronchial epithelial cells and that the ROS level was declined by the ANXA1 over-expression. Oxidative stress is caused by oxidant/antioxidant disorder. The over-generation of ROS will lead to enzymatic and non-enzymatic alterations and the dys-regulation of respiratory airways (40). Our data recorded an increased activity of SOD and Gpx as well as a decreased content of MDA and LDH. Since the imbalance of oxidant/antioxidant enzyme was ameliorated by ANXA1, our research data were in accordance to the results from a previous study, in which the oxidative inactivation and the mucosal wound repair was shown to be promoted by ANXA1 in mucosal wound repair (41).

Activation of the apoptosis pathway is closely related to increased ROS production (42). The cellular caspase-3 activity was enhanced subsequent to being incubated with Bap, following oxidative stress in this study. Moreover, the apoptosis was reduced by the over-expression of ANXA1 via decreasing the expression Bcl-2 and increasing the expression of Bax and cyclin D1. It has been previously suggested that ANXA1 exerted its protective role by repressing the apoptosis. Consistently, it was believed that excessive apoptosis is another mechanism of cell injury due to oxidative stress in airway epithelial cells (43).

PTEN, as a well-known tumor suppressor, can counterbalance the activation of PI3K/Akt (44). The important role of PTEN in resistance to lung cell injury has been demonstrated before (45). FAK is associated with both PTEN and PI3K/Akt based on current knowledge (46). To illustrate the molecular mechanism, the activity of PTEN, FAK, PI3K/Akt was examined. Our data indicated that PTEN and FAK were deactivated by Bap, while their expressions were rescued by the ANXA1 over-expression. A previous study have shown that the antisense oligonucleotides of FAK could depress cell viability (47). Thus, in this study, the anti-apoptotic role of FAK was recognized once more. Furthermore, the hyperactivation of PI3K/Akt caused by Bap was partially blunted by ANXA1. This result was compatible with a publication in which the inactivation of Akt definitively ameliorated ALI and airway remodelling, and retained the integrity of alveolar epithelial cells (17). Nevertheless, researches also pointed out that PI3K-Akt signaling pathway was related to anti-apoptotic functions $(48,49)$. These results appeared to be controversial. However, it is not surprising that PI3K-Akt signaling pathway may exert different roles in the cells, and this may ascribe to different cell type and different study model. In addition, the specific inhibition of PTEN that caused by SF1670 reversed the anti-apoptotic effect of ANXA1, and such a phenomenon suggested that the activation of PTEN was necessary for the protective effect of ANXA1. Moreover, SF1670 reversed the effect of ANXA1 on the expression of PTEN, FAK and p-PI3K/p-Akt. All these data showed that PTEN/FAK/PI3K/Akt pathway was the downstream signaling of ANXA1, and this pathway was participated in Bap induced apoptosis.

Taken together, as the work model shown, the over-expression of ANXA1 suppressed Bap-induced oxidative stress and blunted activation of PI3K/Akt. ROS play important roles in the signal transduction pathways that regulate cell proliferation $(50,51)$. It was implied that the anti-apoptotic role of ANXA1 may ascribe in part to a redox-dependent mechanism (52). Thus, ANXA1 might have the function to prevent bronchial epithelium injury. In addition, if the protective role of ANXA1 can be confirmed by in vivo studies, ANXA1 may have promising therapeutic benefits for the option of asthma.

In summary, our results demonstrated that ANXA1 could sharply reduce the oxidative stress by declining ROS extent and modulating the level of SOD, Gpx, MDA and LDH. Additionally, ANXA1 inhibited apoptosis via regulating the expression of Bcl-2, Bax and cyclin D1. The underlying mechanisms is most likely to be associated with the the activation of PTEN/FAK and the inhibition of p-PI3K/p-Akt. The current study inspired a potential therapeutic strategy for asthma patients.

\section{Acknowledgements}

Not applicable.

\section{Funding}

No funding was received.

\section{Availability of data and materials}

All data generated and/or analyzed during this study are included in this published article.

\section{Authors' contributions}

YC wrote the main manuscript. YC and SY designed the study and performed the experiments. YC performed data analysis. All authors read and approved the manuscript. 


\section{Ethics approval and consent to participate}

Not applicable.

\section{Consent for publication}

Not applicable.

\section{Competing interests}

The authors declare that they have no competing interests.

\section{References}

1. Anandan C, Nurmatov U, van Schayck OC and Sheikh A: Is the prevalence of asthma declining? Systematic review of epidemiological studies. Allergy 65: 152-167, 2010.

2. Worldwide variation in prevalence of symptoms of asthma, allergic rhinoconjunctivitis, and atopic eczema: ISAAC. The International Study of Asthma and Allergies in Childhood (ISAAC) Steering Committee. Lancet 351: 1225-1232, 1998.

3. Kay AB, Phipps S and Robinson DS: A role for eosinophils in airway remodelling in asthma. Trends Immunol 25: 477-482, 2004

4. Capra V and Rovati GE: Rosuvastatin inhibits human airway smooth muscle cells mitogenic response to eicosanoid contractile agents. Pulm Pharmacol Ther 27: 10-16, 2014.

5. Al-Muhsen S, Johnson JR and Hamid Q: Remodeling in asthma. J Allergy Clin Immunol 128: 451-464, 2011.

6. Uno $\mathrm{S}$ and Makishima M: Benzo[a]pyrene toxicity and inflammatory disease. Curr Rheumatol Rev 5: 266-271, 2009.

7. Dong JI and Bozzelli JW: Benzo(a)pyrene levels in several indoor environments with kerosene heaters and wood-burning fireplaces. Chemosphere 18: 1829-1836, 1989.

8. Qamar W, Khan R, Khan AQ, Rehman MU, Lateef A, Tahir M, Ali F and Sultana S: Alleviation of lung injury by glycyrrhizic acid in benzo(a)pyrene exposed rats: Probable role of soluble epoxide hydrolase and thioredoxin reductase. Toxicology 291: 25-31, 2012.

9. Burhans WC and Heintz NH: The cell cycle is a redox cycle: Linking phase-specific targets to cell fate. Free Radic Biol Med 47: 1282-1293, 2009.

10. Michaeloudes C, Sukkar MB, Khorasani NM, Bhavsar PK and Chung KF: TGF- $\beta$ regulates Nox 4, MnSOD and catalase expression, and IL-6 release in airway smooth muscle cells. Am J Physiol Lung Cell Mol Physiol 300: L295-L304, 2011.

11. Kirkham P and Rahman I: Oxidative stress in asthma and COPD: Antioxidants as a therapeutic strategy. Pharmacol Ther 111: 476-494, 2006.

12. Schieber M and Chandel NS: ROS function in redox signaling and oxidative stress. Curr Biol 24: R453-R462, 2014.

13. Kannan K and Jain SK: Oxidative stress and apoptosis. Pathophysiology 7: 153-163, 2000.

14. Keniry M and Parsons R: The role of PTEN signaling perturbations in cancer and in targeted therapy. Oncogene 27: 5477-5485, 2008.

15. Frisch SM, Vuori K, Ruoslahti E and Chan-Hui PY: Control of adhesion-dependent cell survival by focal adhesion kinase. J Cell Biol 134: 793-799, 1996.

16. Tamura M, Gu J, Matsumoto K, Aota S, Parsons R and Yamada KM: Inhibition of cell migration, spreading, and focal adhesions by tumor suppressor PTEN. Science 280: 1614-1617, 1998.

17. Miyoshi K, Yanagi S, Kawahara K, Nishio M, Tsubouchi H, Imazu Y, Koshida R, Matsumoto N, Taguchi A, Yamashita S, et al: Epithelial Pten controls acute lung injury and fibrosis by regulating alveolar epithelial cell integrity. Am J Respir Crit Care Med 187: 262-275, 2013.

18. Maehama Tand Dixon JE: The tumor suppressor,PTEN/MMAC1, dephosphorylates the lipid second messenger, phosphatidylinositol 3,4,5-trisphosphate. J Biol Chem 273: 13375-13378, 1998.

19. Schaller MD: Signaling through the focal adhesion kinase. Soc Gen Physiol Ser 52: 241-255, 1997.

20. Levkau B, Herren B, Koyama H, Ross R and Raines EW: Caspase-mediated cleavage of focal adhesion kinase pp125FAK and disassembly of focal adhesions in human endothelial cell apoptosis. J Exp Med 187: 579-586, 1998.
21. Sonoda Y, Watanabe S, Matsumoto Y, Aizu-Yokota E and Kasahara T: FAK is the upstream signal protein of the phosphatidylinositol 3-kinase-Akt survival pathway in hydrogen peroxide-induced apoptosis of a human glioblastoma cell line. J Biol Chem 274: 10566-10570, 1999.

22. Goulding NJ, Godolphin JL, Sampson MB, Maddison PJ and Flower RJ: Hydrocortisone induces lipocortin 1 production by peripheral blood mononuclear cells in vivo in man. Biochem Soc Trans 18: 306-307, 1990.

23. Chatterjee BE, Yona S, Rosignoli G, Young RE, Nourshargh S, Flower RJ and Perretti M: Annexin 1-deficient neutrophils exhibit enhanced transmigration in vivo and increased responsiveness in vitro. J Leukoc Biol 78: 639-646, 2005.

24. Perretti M, Ahluwalia A, Harris JG, Goulding NJ and Flower RJ: Lipocortin-1 fragments inhibit neutrophil accumulation and neutrophil-dependent edema in the mouse. A qualitative comparison with an anti-CD11b monoclonal antibody. J Immunol 151: 4306-4314, 1993.

25. D'Amico M, Di Filippo C, La M, Solito E, McLean PG, Flower RJ, Oliani SM and Perretti M: Lipocortin 1 reduces myocardial ischemia-reperfusion injury by affecting local leukocyte recruitment. FASEB J 14: 1867-1869, 2000.

26. La M, D'Amico M, Bandiera S, Di Filippo C, Oliani SM, Gavins FN, Flower RJ and Perretti M: Annexin 1 peptides protect against experimental myocardial ischemia-reperfusion: Analysis of their mechanism of action. FASEB J 15: 2247-2256, 2001.

27. Martin GR, Perretti M, Flower RJ and Wallace JL: Annexin-1 modulates repair of gastric mucosal injury. Am J Physiol Gastrointest Liver Physiol 294: G764-G769, 2008.

28. Li Y, Prasad A, Jia Y, Roy SG, Loison F, Mondal S, Kocjan P, Silberstein LE, Ding S and Luo HR: Pre-treatment with phosphatase and tensin homolog deleted on chromosome 10 (PTEN) inhibitor SF1670 augments the efficacy of granulocyte transfusion in a clinically relevant mouse model. Blood: 117: 6702-6713, 2011.

29. Livak KJ and Schmittgen TD: Analysis of relative gene expression data using real-time quantitative PCR and the 2(-Delta Delta C(T)) method. Methods 25: 402-408, 2001

30. Feng L, Le J, Lan Y, Pang W and Wang C: The role of ROS in human bronchial epithelial cell injury caused by gunpowder smoke. Lab Immun Clin Med 17: 27-29, 2010.

31. Chandra J, Samali A and Orrenius S: Triggering and modulation of apoptosis by oxidative stress. Free Radic Biol Med 29: $323-333,2000$

32. Sedlak TW, Oltvai ZN, Yang E, Wang K, Boise LH, Thompson CB and Korsmeyer SJ: Multiple Bcl-2 family members demonstrate selective dimerizations with Bax. Proc Natl Acad Sci USA 92: 7834-7838, 1995.

33. Albanese C, D'Amico M, Reutens AT, Fu M, Watanabe G, Lee RJ, Kitsis RN, Henglein B, Avantaggiati M, Somasundaram K, et al: Activation of the cyclin D1 gene by the E1A-associated protein p300 through AP-1 inhibits cellular apoptosis. J Biol Chem 274: 34186-34195, 1999.

34. Xia H, Diebold D, Nho R, Perlman D, Kleidon J, Kahm J, Avdulov S, Peterson M, Nerva J, Bitterman P and Henke C: Pathological integrin signaling enhances proliferation of primary lung fibroblasts from patients with idiopathic pulmonary fibrosis. J Exp Med 205: 1659-1672, 2008.

35. White ES, Atrasz RG, Hu B, Phan SH, Stambolic V, Mak TW, Hogaboam CM, Flaherty KR, Martinez FJ, Kontos CD and Toews GB: Negative regulation of myofibroblast differentiation by PTEN (Phosphatase and Tensin Homolog Deleted on chromosome 10). Am J Respir Crit Care Med 173: 112-121, 2006.

36. Sonoda Y, Matsumoto Y, Funakoshi M, Yamamoto D, Hanks SK and Kasahara T: Anti-apoptotic role of Focal Adhesion Kinase (FAK). Induction of inhibitor-of-apoptosis proteins and apoptosis suppression by the overexpression of FAK in a human leukemic cell line, HL-60. J Biol Chem 275: 16309-16315, 2000.

37. Alexandrov K, Rojas M and Satarug S: The critical DNA damage by benzo(a)pyrene in lung tissues of smokers and approaches to preventing its formation. Toxicol Lett 198: 63-68, 2010.

38. Podechard N, Lecureur V, Le Ferrec E, Guenon I, Sparfel L, Gilot D, Gordon JR, Lagente V and Fardel O: Interleukin-8 induction by the environmental contaminant benzo(a)pyrene is aryl hydrocarbon receptor-dependent and leads to lung inflammation. Toxicol Lett 177: 130-137, 2008.

39. Babbin BA, Laukoetter MG,Nava P,Koch S, Lee WY,Capaldo CT, Peatman E, Severson EA, Flower RJ, Perretti M, et al: Annexin A1 regulates intestinal mucosal injury, inflammation, and repair. J Immunol 181: 5035-5044, 2008. 
40. Nadeem A, Masood A and Siddiqui N: Oxidant-antioxidant imbalance in asthma: Scientific evidence, epidemiological data and possible therapeutic options. Ther Adv Respir Dis 2: 215-235, 2008.

41. Leoni G, Alam A, Neumann PA, Lambeth JD, Cheng G, McCoy J, Hilgarth RS, Kundu K, Murthy N, Kusters D, et al: Annexin A1, formyl peptide receptor, and NOX1 orchestrate epithelial repair. J Clin Invest 123: 443-454, 2013.

42. Park EJ, Yi J, Chung KH, Ryu DY, Choi J and Park K: Oxidative stress and apoptosis induced by titanium dioxide nanoparticles in cultured BEAS-2B cells. Toxicol Lett 180: 222-229, 2008.

43. Truong-Tran AQ, Grosser D, Ruffin RE, Murgia C and Zalewski PD: Apoptosis in the normal and inflamed airway epithelium: Role of zinc in epithelial protection and procaspase-3 regulation. Biochem Pharmacol 66: 1459-1468, 2003.

44. Stambolic V, Suzuki A, de la Pompa JL, Brothers GM, Mirtsos C Sasaki T, Ruland J, Penninger JM, Siderovski DP and Mak TW: Negative regulation of $\mathrm{PKB} / \mathrm{Akt}$-dependent cell survival by the tumor suppressor PTEN. Cell 95: 29-39, 1998.

45. Tiozzo C, De Langhe S, Yu M, Londhe VA, Carraro G, Li M, Li C, Xing Y, Anderson S, Borok Z, et al: Deletion of Pten expands lung epithelial progenitor pools and confers resistance to airway injury. Am J Respir Crit Care Med 180: 701-712, 2009.

46. You D, Xin J, Volk A, Wei W, Schmidt R, Scurti G, Nand S, Breuer EK, Kuo PC, Breslin P, et al: FAK mediates a compensatory survival signal parallel to PI3K-AKT in PTEN-null T-ALL cells. Cell Rep 10: 2055-2068, 2015.

47. Sonoda Y, Kasahara T, Yokota-Aizu E, Ueno M and Watanabe S: A suppressive role of p125FAK protein tyrosine kinase in hydrogen peroxide-induced apoptosis of T98G cells. Biochem Biophys Res Commun 241: 769-774, 1997.
48. Wu W: DLX4 homeoprotein promotes PI3K/Akt anti-apoptosis pathway in ER negative breast cancer cells through upregulation of VEGFA. Dissert Thes-Gradwork, 2010.

49. Wang XQ, Yao RQ, Liu X, Huang JJ, Qi DS and Yang LH: Quercetin protects oligodendrocyte precursor cells from oxygen/glucose deprivation injury in vitro via the activation of the PI3K/Akt signaling pathway. Brain Res Bull 86: 277-284, 2011.

50. Qin S, Inazu T, Takata M, Kurosaki T, Homma $\mathrm{Y}$ and Yamamura H: Cooperation of tyrosine kinases p72syk and p53/56lyn regulates calcium mobilization in chicken B cell oxidant stress signaling. Eur J Biochem 236: 443-449, 1996.

51. Suzuki Y, Ohsugi K and Ono Y: Oxidative stress triggers tyrosine phosphorylation in B cells through a redox- and inflammatory cytokine-sensitive mechanism. Immunology 87 : 396-401, 1996.

52. Sturrock A, Huecksteadt TP, Norman K, Sanders K, Murphy TM, Chitano P, Wilson K, Hoidal JR and Kennedy TP: Nox4 mediates TGF-beta1-induced retinoblastoma protein phosphorylation, proliferation, and hypertrophy in human airway smooth muscle cells. Am J Physiol Lung Cell Mol Physiol 292: L1543-L1555, 2007. International (CC BY-NC-ND 4.0) License. 